\title{
Editorial
}

\section{Mathematical and Computational Analyses of Flow and Transport Phenomena}

\author{
Shuyu Sun, ${ }^{1}$ Mohamed Fathy El-Amin, ${ }^{2}$ and Jianzhong Lin $^{3}$ \\ ${ }^{1}$ Computational Transport Phenomena Laboratory, King Abdullah University of Science and Technology, \\ Thuwal 23955-6900, Saudi Arabia \\ ${ }^{2}$ Department of Mathematics, Aswan University, Aswan 81528, Egypt \\ ${ }^{3}$ Faculty of Engineering Zhejiang University, Zhejiang, China
}

Correspondence should be addressed to Shuyu Sun; shuyu.sun@kaust.edu.sa

Received 7 April 2014; Accepted 7 April 2014; Published 8 May 2014

Copyright (C) 2014 Shuyu Sun et al. This is an open access article distributed under the Creative Commons Attribution License, which permits unrestricted use, distribution, and reproduction in any medium, provided the original work is properly cited.

\section{Introduction}

In this special issue, a number of papers have been accepted for publication. The special issue concerns with theoretical investigation and mathematical analysis that are very important for all scientific, engineering, and environmental applications. From mathematical modeling to computational analysis and all the way to developing analytical and numerical solutions, studying solutions properties, and so forth, the theoretical, mathematical, and computational analyses are indispensable bases. Rapid progress has been seen in the analysis of flow and transport phenomena especially in the recent years because of the significance of flow and transport to science and engineering.

\section{Overview of Work Presented in This Special Issue}

The list of papers published in this issue covers a wide range of applications using different approaches and analyses, and it may be divided into five groups as follows.

The first group of papers consists of nine papers that address various issues in the area of particles/nanoparticles suspensions flow that are used in different applications. Z. You et al. have studied a long-term deep bed filtration in porous media with size exclusion particle capture mechanism in the paper entitled "Exact solution for long-term size exclusion suspension-colloidal transport in porous media." On the other hand, E. H. Aly and A. Ebaid have introduced a direct and effective approach to obtain the exact analytical solution for the nanoparticles-water flow over an isothermal stretching sheet with the effect of the slip model in the paper entitled "Exact analytical solution for suction and injection flow with thermal enhancement of five nanofluids over an isothermal stretching sheet with effect of the slip model: a comparative study." In another paper entitled "The flow and heat transfer of a nanofluid past a stretching/shrinking sheet with a convective boundary condition," S. Mansur and A. Ishak have studied the boundary layer flow of a nanofluid past a stretching/shrinking sheet with a convective boundary condition. Moreover, H. Qing and X. Mingliang have proposed a model of fundamental aspects of the Taylor-series expansion method of moment (TEMOM) to describe the aerosol population balance equation due to Brownian coagulation in the continuum regime in the paper entitled "The fundamental aspects of TEMOM model for particle coagulation due to Brownian motion-part ii: in the continuum regime." $\mathrm{R}$. Wang has presented numerical investigations on particle trapping techniques by using intrinsic hydrodynamic effects in an expansion-contraction microfluidic device in the paper entitled "Hydrodynamic trapping of particles in an expansioncontraction microfluidic device." L.-Z. Huang and D.-M. Nie in the article entitled "Lattice Boltzmann simulation of collision between 2D circular particles suspension in Couette flow" simulated the collision between 2D circular particles suspension in Couette flow by using multiple relaxation timebased lattice Boltzmann and direct forcing/fictitious domain method. In the paper entitled "Modeling and numerical 
analysis of the solid particle erosion in curved ducts," K. Sun et al. presented a modeling and computational study on particle erosion in curved ducts. In another paper, $\mathrm{M}$. H. Alawi et al. have introduced a mathematical model to describe the fineparticles transport carried by a two-phase flow in a porous medium to describe the formation damage of asphalt-paved roads in the paper entitled "Modeling and simulation of flow and formation damage of asphalt-paved roads." Finally, F. Yuan and F. Gan have used the method of moments to predict the evolution of aerosol particles in the rainfall process in the paper entitled "Evolution of aerosol particles in the rainfall process via method of moments." In the paper entitled "Direct numerical simulation of concentration and orientation distribution of fibers in a mixing layer" by K. Zhou et al., the concentration and orientation of suspended fibers in a mixing layer are investigated numerically.

The second group is concerned with reservoir modeling and simulation. S. Borazjani et al. presented the paper entitled "Exact solution for non-self-similar wave-interaction problem during two-phase four-component flow in porous media." Analytical solutions for one-dimensional two-phase multicomponent flows in porous media describe processes of enhanced oil recovery, environmental flows of waste disposal, and contaminant propagation in subterranean reservoirs and water management in aquifers. In the paper entitled "New scheme of finite difference heterogeneous multiscale method to solve saturated flow in porous media," F. Chen and L. Ren have constructed a finite difference scheme, namely, the development of the finite difference heterogeneous multiscale method (FDHMM), for simulating saturated water flow in random porous media. J. Xu et al. in their paper " $A$ direct Eulerian GRP scheme for the prediction of gas-liquid two-phase flow in HTHP transient wells" have introduced a dimensional splitting technique with Eulerian generalized Riemann problem (GRP) scheme to solve coupled system model of partial differential equations which concerns with the variation of the pressure and temperature, velocity, and density at different times and depths in high temperature-high pressure (HTHP) gas-liquid two-phase flow wells. In addition to flow simulation in reservoir, reservoir sedimentation is also considered in this issue. The basic factors influencing the density of sediments deposited in reservoirs are discussed, and uncertainties in reservoir sedimentation have been determined using the Delta method by F. Imanshoar et al. in the paper entitled "Reservoir sedimentation based on uncertainty analysis." Also, the paper of L. Gan and J. Xu, "Retrofitting transportation network using a fuzzy random multiobjective bilevel model to hedge against seismic risk," focuses on the problem of hedging against seismic risk through the retrofit of transportation systems in large-scale construction projects.

The third group, which consists of two papers, is concerned with some biological applications. In the first paper entitled "A new method of moments for the bimodal particle system in the Stokes regime," Y.-H. Liu and Z.-Q. Yin studied the particle system in the Stokes regime with a bimodal distribution. A. Y. Tang and N. Amin have reviewed some numerical approaches to solve fluid structure interaction problems in blood flow in the paper entitled "Some numerical approaches to solve fluid structure interaction problems in blood flow."

The fourth group focuses on some heat transfer in fluid flow problems. M. Goodarzi et al. have introduced a comparison study for the problem of natural convection heat transfer inside cavities and enclosures in the paper entitled "Comparison of the finite volume and lattice Boltzmann methods for solving natural convection heat transfer problems inside cavities and enclosures." In the paper entitled "Revisiting Blasius flow by fixed point method," D. Xu et al. have used the fixed-point method to resolve the Blasius problem.

The last group is related to flow and transport applications in electronic devices. Numerical simulations for the melt flow under the influence of control devices in a T-type twostrand bloom caster tundish are presented by Z. He et al. in the paper entitled "Numerical modeling of the fluid flow in continuous casting tundish with different control devices." The fluid-driven efficiency of the micropump based on induced charge electroosmotic was studied numerically by K. Zhang et al. in the paper entitled "Design of T-shaped micropump based on induced charge electroosmotic."

\section{Conclusions}

This special issue presents and highlights new applications and new challenges in five different important research areas of flow and transport. This special issue is not intended to be an exhaustive collection nor a survey of all of the current trends in flow and transport research; many additional significant research areas of flow and transport still exist and remain to be explored, but multidisciplinary research effort is a clear trend.

\section{Acknowledgments}

The authors would like to thank the participants of the special issue for their inspiring contributions and the anonymous reviewers for their diligent work, which led to the high quality of the special issue. The lead guest editor S. Sun would like to acknowledge KAUST Faculty Baseline Research Fund (BRF) for supporting his research in flow and transport.

Shuyu Sun Mohamed Fathy El-Amin Jianzhong Lin 


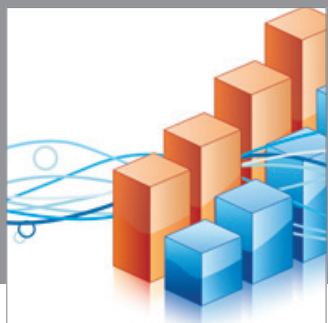

Advances in

Operations Research

mansans

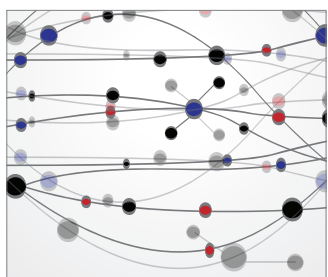

The Scientific World Journal
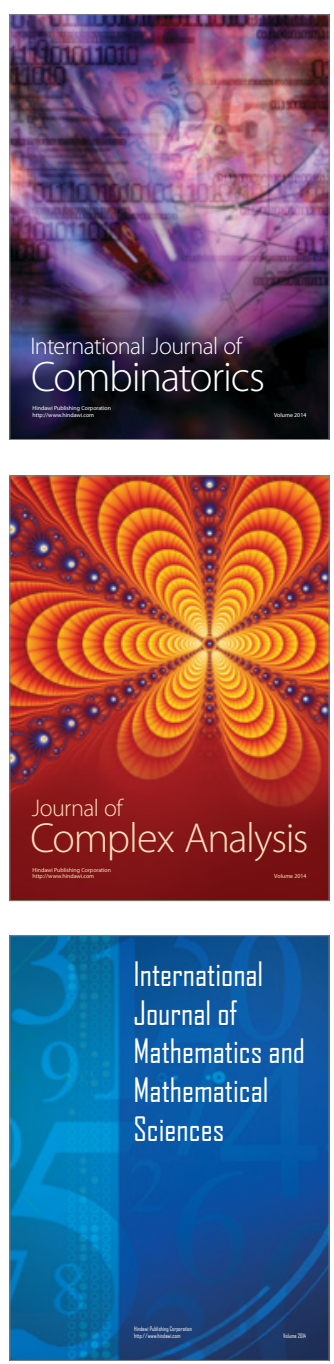
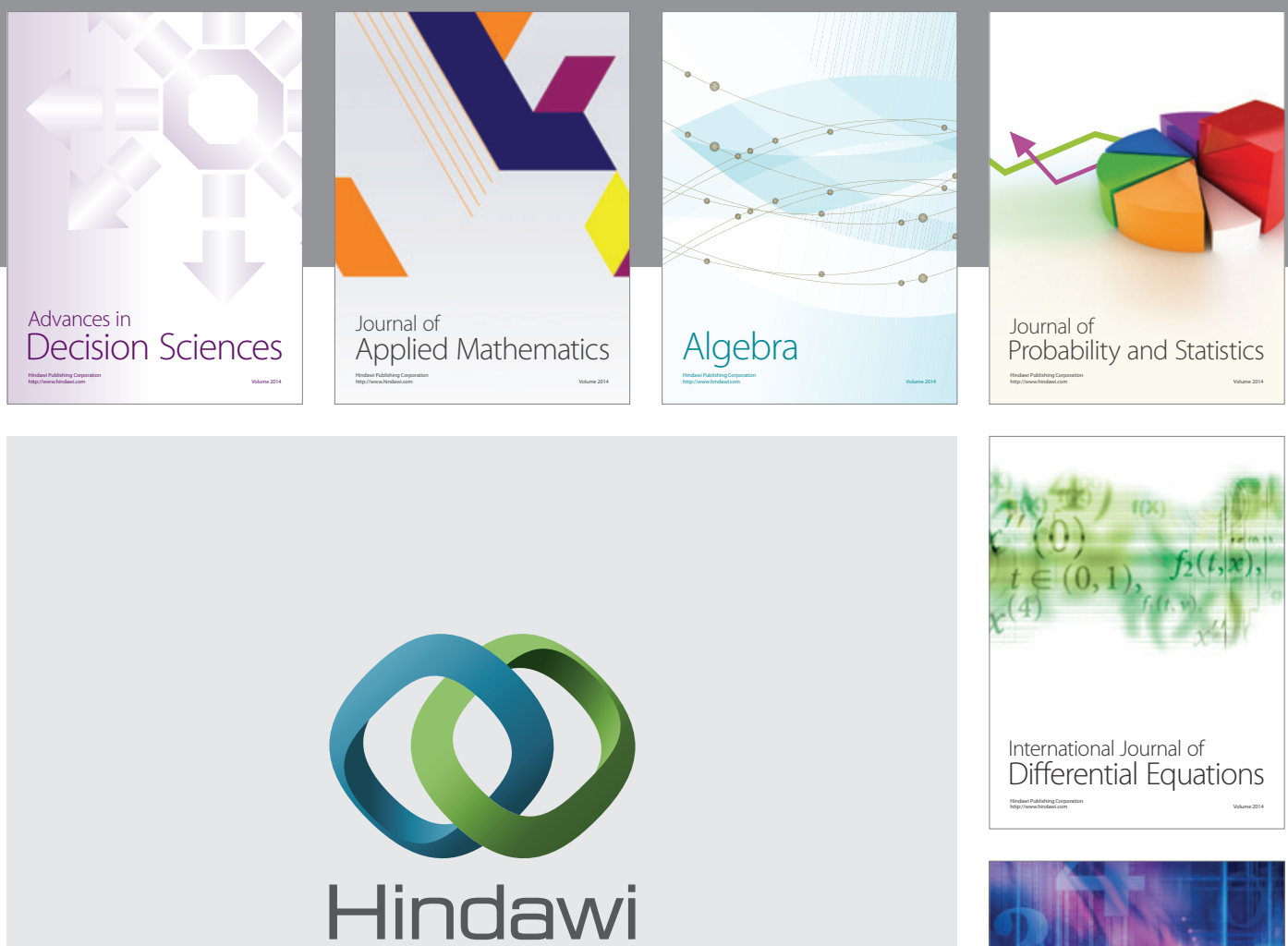

Submit your manuscripts at http://www.hindawi.com
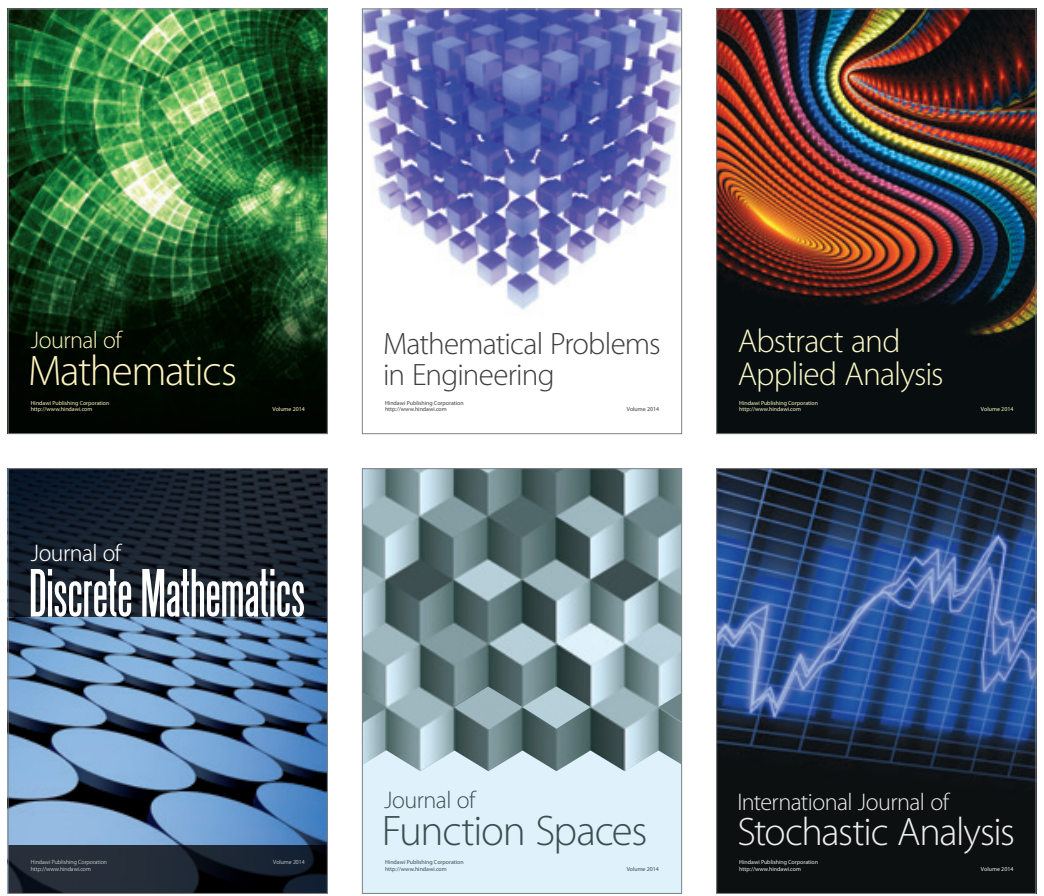

Journal of

Function Spaces

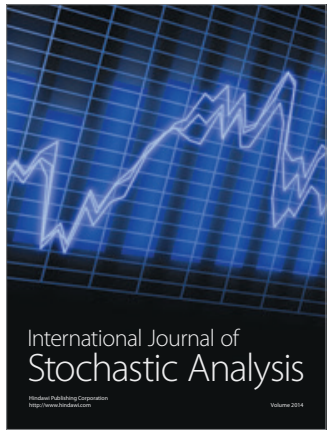

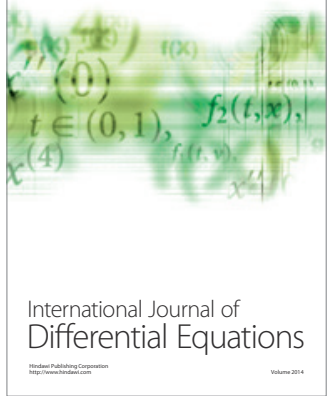
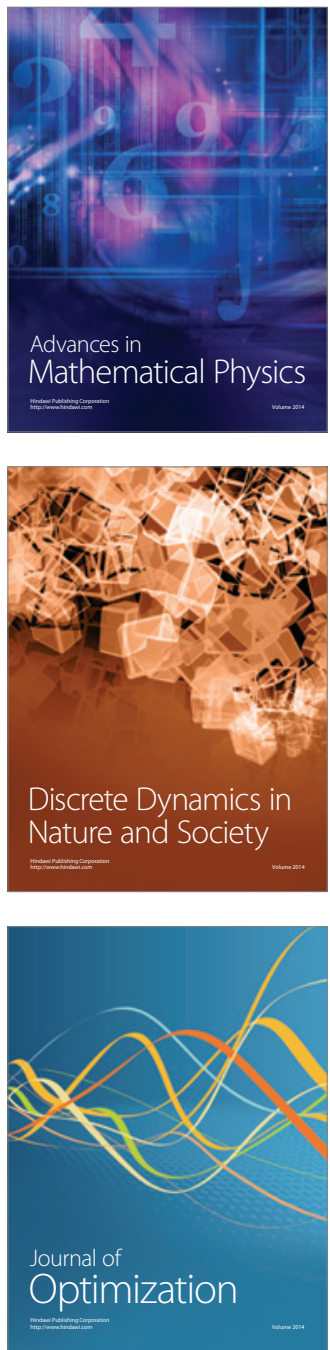